\title{
A Comparison of Gait Characteristics Between Older Women with and Without Peripheral Neuropathy in Standard and Challenging Environments
}

\author{
James K. Richardson, MD, Sibylle B. Thies, MS, Trina K. DeMott, MS, PT, \\ and James A. Ashton-Miller, PhD
}

OBJECTIVES: To compare gait patterns in older women with and without peripheral neuropathy (PN) in standard (smooth surface, normal lighting) and challenging environments (CE) (irregular surface, low lighting).

DESIGN: Observational, controlled study of 24 subjects.

SETTING: Biomechanical research laboratory.

PARTICIPANTS: Twenty-four older women, 12 with PN and 12 without $\mathrm{PN}$ (mean age \pm standard deviation $=67.1 \pm$ 7.9 and $70.2 \pm 4.3$, respectively).

MEASUREMENTS: Gait parameters and, in the $12 \mathrm{PN}$ subjects, neuropathy severity.

RESULTS: The CE was associated with increases in step width, step-width variability, step-width range, step widthto-step length ratio, step time and step-time variability, and decreases in step length and speed. The PN subjects demonstrated a greater step width-to-step length ratio and step time and shorter step length and slower speed than the control subjects. In adapting to the CE, the PN subjects demonstrated greater increases in step width-to-step length ratio and step-time variability and a greater decrease in step length than did the control subjects. In the standard environment, only one gait parameter correlated with $\mathrm{PN}$ severity, whereas in the $\mathrm{CE}$, four gait parameters did so.

CONCLUSION: The subjects demonstrated a gait that was slower, less efficient, and more variable temporally and in the frontal plane in the CE. Control and PN subjects demonstrated similar variability in medial-lateral step placement in the CE but at the cost of speed and efficiency for the PN subjects. Because the CE magnified gait differences between the two groups of subjects and caused gait changes in the PN subjects that correlated with PN severity, the $\mathrm{CE}$ may offer improved resolution for detecting gait abnormalities. J Am Geriatr Soc 52:1532-1537, 2004.

Key words: gait; balance; neuropathy

From the Departments of Physical Medicine and Rehabilitation and Mechanical Engineering, University of Michigan, Ann Arbor, Michigan.

Address correspondence to Dr. James K. Richardson, Associate Professor, University of Michigan, Department of Physical Medicine and Rehabilitation, Medical Professional Bldg. D5200, Ann Arbor, MI 48109. E-mail: jkrich@umich.edu
B ecause longer axons are usually more vulnerable to disease, a diffuse peripheral neuropathy $(\mathrm{PN})$ typically reduces sensorimotor function in a distal-to-proximal pattern, leading to reduced sensation and motor impairments in the feet and ankles. ${ }^{1}$ Given the importance of somatosensory input to the maintenance of balance, ${ }^{2}$ it is not surprising that older persons with $\mathrm{PN}$ are at a markedly increased risk for injurious falls. ${ }^{3,4}$ Furthermore the prevalence of PN is high, estimated to affect slightly more than $20 \%$ of persons aged 65 to 75 in the United States. ${ }^{5}$ This prevalence appears to be increasing, likely driven by increasing rates of type II diabetes mellitus and glucose intolerance. $^{6}$

The study of gait is of interest because a large proportion of falls in older persons, with and without PN, occur during ambulation. ${ }^{3,7}$ Differences between neuropathic and normal gait identified under ideal circumstances (level surface with good lighting) include decreased walking speed, increased frontal plane motion at the ankle, ${ }^{8}$ increased time in double support, ${ }^{9}$ and substitution of hip flexion for plan$\operatorname{tar}$ flexion..$^{10}$ Despite these differences, PN subjects have not been found to be unstable while walking on a level surface with good lighting. Underscoring this point, one study found that PN patients achieved better dynamic upper body stability than did control subjects and concluded that falls by patients with PN were "likely due to their inability to develop and execute appropriate avoidance and/ or response strategies when faced with unexpected obstacles or large-scale perturbations during locomotion." 11 This conclusion agrees with the authors' clinical experience.

Therefore, gait patterns of older persons with $\mathrm{PN}$ in an environment that causes unexpected perturbations are of interest. In this study, two groups of older women, one group with and the other without PN, were observed walking on a flat surface with high lighting (standard environment (SE)) and an irregularly contoured surface with low lighting (challenging environment $(\mathrm{CE})$ ). Lower extremity and trunk positions were continuously recorded so that gait parameters could be determined. Gait parameters and their clinical relevance included step-width variability and stepwidth range because of their association with frontal plane control; ${ }^{12}$ step width-to-step length ratio because of its 
relationship to gait efficiency; ${ }^{13}$ step length and step time, which determine speed and are of functional importance in time-contingent activities such as crossing a street with a light; ${ }^{14}$ and step-time variability because of its relationship to falls. ${ }^{15}$ It was hypothesized that the CE and the presence of PN would lead to increasing gait variability, both temporally and in the frontal plane, and decreased speed and efficiency.

\section{METHODS}

\section{Subjects}

PN subjects were recruited from the University of Michigan Electrodiagnostic Laboratory and Physical Medicine and Rehabilitation Outpatient Orthotics and Prosthetics Clinic. All patients underwent history, physical examination, and electrodiagnostic testing. History and physical examination focused on the identification of central neurological or musculoskeletal abnormalities. The University of Michigan institutional review board approved the project, and all subjects gave written informed consent.

Inclusion criteria for $\mathrm{PN}$ subjects were: age between 50 and 85 , symptoms consistent with PN, ability to speak and understand English, ability to ambulate household distances without an assistive device, physical examination consistent with PN (absent or decreased Achilles reflexes, decreased distal lower extremity sensation (vibration, pinprick and light touch) which improved proximally) and electrodiagnostic evidence of a diffuse, primarily axonal polyneuropathy as evidenced by an abnormal sural response (absent or amplitude $<6 \mu \mathrm{V}$ ) and peroneal or tibial motor responses (absent or amplitude $<2.0$ or $3.0 \mu \mathrm{V}$, respectively). The subjects were also evaluated using the Michigan Diabetes Neuropathy Score (MDNS), a 0- to 46point scale (higher score reflecting more severe $\mathrm{PN}$ ) that correlates well with more extensive neuropathy staging scales. ${ }^{16}$

Exclusion criteria were weight greater than $300 \mathrm{lbs}$ $(135.7 \mathrm{~kg})$, history of balance disorder unrelated to PN, evidence on physical examination of vestibular or central neurological dysfunction, or a musculoskeletal abnormality such as severe scoliosis or amputation.

The control subjects were recruited through the University of Michigan Older Americans Independence Center Research Participant Program. The inclusion/exclusion criteria for the control subjects were the same as those for the PN subjects, with the exception that the control subjects had no symptoms or signs of PN. The control subjects underwent the same screening history and physical examination as the PN subjects but did not undergo electrodiagnostic testing.

\section{Subject Preparation and Experimental Apparatus}

The subjects were placed in a safety harness that was attached by climbing rope to an overhead track (Figure 1). The cords were adjusted to prevent the knees from coming into contact with the floor when the subject hung unsupported. Subjects wore standard, flat-soled athletic shoes supplied by the laboratory. For all trials, subjects were instructed to walk at their own pace, as if they were "walking to mail a letter." The SE included a flat, linoleum tile walking
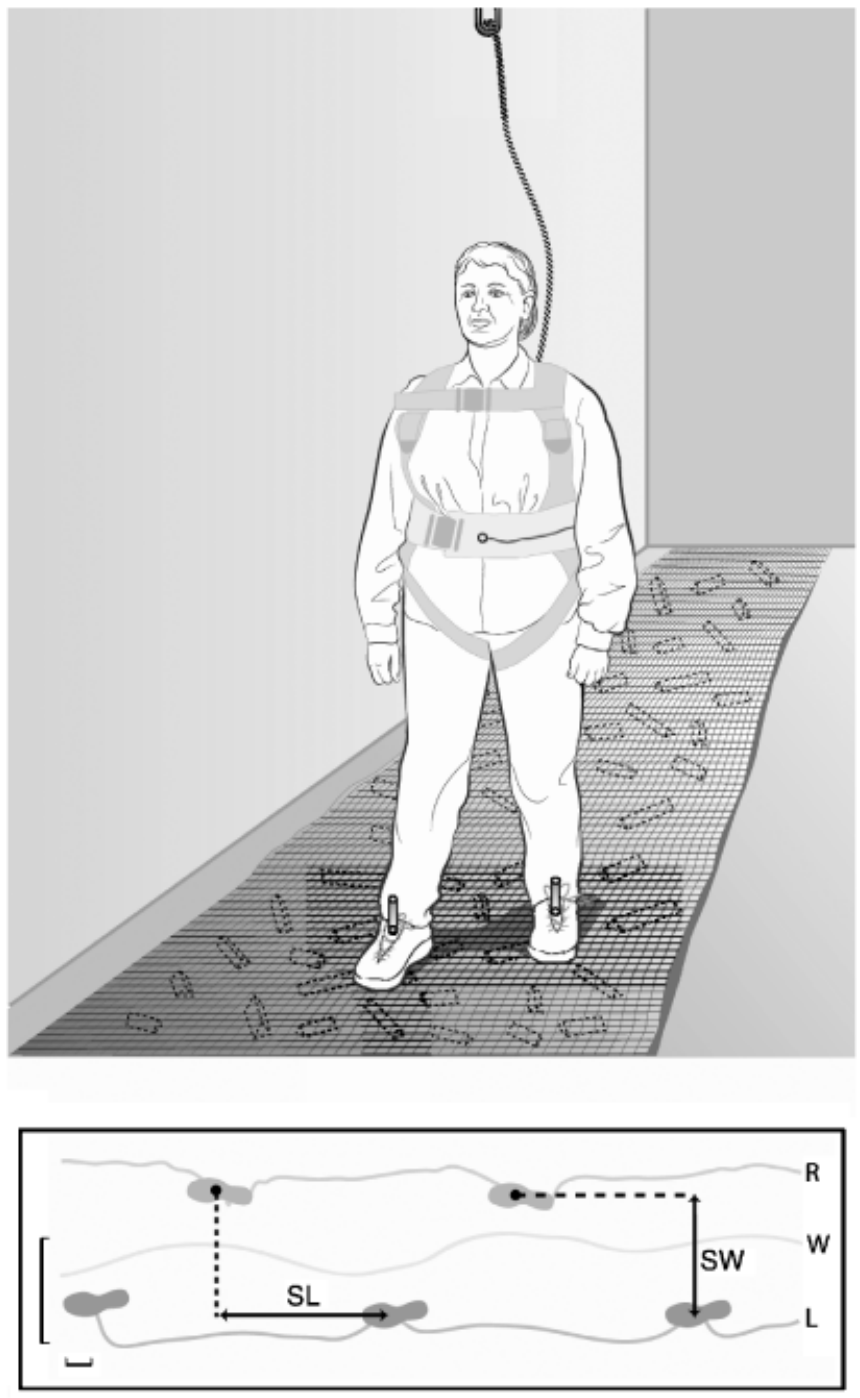

Figure 1. Subject preparation and experimental apparatus. Top illustration: Schematic of irregular walkway used during the experiment. Wooden prisms are oriented randomly beneath an industrial carpeted surface. Waist and ankle electronic markers are shown. Lower illustration: Sample data for one trial are shown. Optoelectronic position data for the right $(\mathrm{R})$ and left $(\mathrm{L})$ ankle markers were used for calculation of step width (SW) and step length (SL). The waist marker (W) trajectory is also shown. Kinematic data were scaled according to the $10-\mathrm{cm}$ bars in the lower left corner.

surface and lighting maintained at more than 900 lux by overhead light. The CE included an irregularly contoured walking surface and dimmed room lights $(<50$ lux $)$. The walkway $(1.5 \times 10 \mathrm{~m})$ was created by randomly arranging prism-shaped pieces of wood $(1.5 \mathrm{~cm}$ high, $3.5 \mathrm{~cm}$ wide, $6-$ $16 \mathrm{~cm}$ long) beneath dark industrial carpet (Figure 1). Two optoelectronic markers (infrared-emitting diodes) were placed $5 \mathrm{~cm}$ apart on a malleable aluminum strip $(10 \mathrm{~cm} \times$ $1.5 \mathrm{~cm}$ ) inserted under the tongue of each shoe. The top marker was located anterior to the center of the malleoli (estimated to be the center of the tibiotalar joint). A waist marker was placed on a belt in the midline at the level of the umbilicus. Two foot switches, each a force-sensing resistor, 
were placed underneath the insole of each shoe. One switch was placed under the first metatarsophalangeal joint and the other beneath the calcaneus. Double support was defined as the period of time in the gait cycle during which at least one switch inside each shoe was activated. Step width, step length, and average speed were measured at $100 \mathrm{~Hz}$ using an optoelectronic camera system (Optotrak 3020, Northern Digital Corp., Waterloo, Ontario) toward which the subject walked within the boundaries of the walkway.

\section{Gait/Data Analysis}

The kinematic and force data were processed using a custom algorithm written in MATLAB to quantify step width, step length, and walking speed. Speed was calculated by taking the time derivative of the waist marker during what was defined as the "comfortable gait speed" interval. This interval was found by excluding data taken when the waist velocity was less then $85 \%$ of the maximum velocity for that trial. This was done to eliminate steps taken while the subject accelerated to and decelerated from comfortable gait speed. Similarly, the other gait parameters were only included in the analysis during this interval. Step time was determined by calculating the time elapsed between closure of the right and left metatarsal foot switch during comfortable gait speed. Step width and step length were defined as shown in Figure 1.

\section{Statistical Analysis of Gait Parameters}

SPSS version 11.0 (SPSS Inc. Chicago, IL) was used for all analyses. Repeated measures analysis of variance (ANOVA) was used to identify subject group and environment effects, and subject group-by-environment interactions. Age and body mass index (BMI) (weight in $\mathrm{kg} /$ height in $\mathrm{m}^{2}$ ) were used as covariates. Environment-by-age and environmentby-BMI interactions were also explored. Descriptive statistics of gait parameters for both subject groups under each environmental condition were generated. Step-width range was determined by subtracting the smallest from the largest step width for each subject in each environment. The standard deviations of step width and step time were used as measures of step-width and step-time variability. Twotailed paired $t$ tests were used to compare differences in mean gait parameters within each subject group in the two environmental conditions. Standard two-tailed $t$ tests were used to compare group differences in demographic data and mean gait parameters when walking under the same environmental condition. Pearson correlations were used to explore relationships between clinical PN severity, as determined by MDNS score, and gait parameters within the 12 PN subjects. To adjust for multiple comparisons of means a $P$-value $<.006$ was considered significant and a $P$ value $\geq .006$ and $<.0125$ was considered to be a trend for the $t$ tests and repeated measures ANOVA.

\section{RESULTS}

\section{Subjects}

The PN and control subjects were of similar age (mean \pm standard deviation $67.1 \pm 7.9$ vs $70.2 \pm 4.3, P=.238)$ and BMI $\left(30.3 \pm 9.5\right.$ vs $\left.30.3 \pm 12.5 \mathrm{~kg} / \mathrm{m}^{2}, P=.993\right)$. As anticipated, the PN subjects demonstrated higher MDNS scores
$(17.7 \pm 5.7$ vs $1.4 \pm 1.5, P<.001)$. The causes of $\mathrm{PN}$ in the PN subjects were diabetes mellitus $(\mathrm{n}=5)$, connective tissue disease $(n=4)$, past exposure to chemotherapy $(n=1)$ and idiopathic $(\mathrm{n}=2)$.

\section{The Effect of Environment}

Repeated measures ANOVA showed that environment had a significant effect on all gait parameters. The CE was associated with increases in step width, step-width variability, step-width range, step width-to-step length ratio, step time, step-time variability, and decreases in step length and speed (Figure 2). Therefore, the subjects demonstrated a slower, wider-based, and more variable gait in the CE.

Direct comparisons of gait parameters under the two environmental conditions, along with accompanying paired $t$ test results, are shown in Table 1 . The effect of the CE was to make the gait of the PN subjects more temporally variable, wider-based, and slower - the last due to decreased step length and increased step time. In general, the control subjects demonstrated similar, but less marked, changes in gait parameters in the CE, but unlike the PN subjects, the control subjects did not decrease their step length or increase step width in the CE. As a result, the control subjects did not demonstrate an increase in step width-to-step length ratio or decrease in speed in the CE.

\section{The Effect of Subject Group}

Multivariate analysis showed that subject group affected all gait parameters except step width, step-width range, and variability (Figure 2). The PN subjects demonstrated significantly increased step width-to-step length ratio, decreased step length and speed, and increased step time and step-time variability (trend). Therefore the PN subjects demonstrated a gait that was slower due to decreased step length and more temporally variable. No significant age or BMI effects were identified.

Direct comparisons of gait parameters between subject groups, and the results of the corresponding $t$ tests, are presented in Table 1. Group differences identified are similar to those noted with multivariate analysis, with the exception that, in the SE, PN subjects did not show differences from the controls in step-time variability. With regard to step-width variability and range, there were no group differences in either environment, and in the CE, the stepwidth variability values were nearly equal $(40.7 \pm 10.1$ vs $39.8 \pm 10.9 \mathrm{~mm})$.

\section{Subject Group-by-Environment Interactions}

Significant subject group-by-environment interactions for step width-to-step length ratio, step length, and step-time variability (trend) were identified (Figure 2). More specifically, in adapting to the $\mathrm{CE}$ the $\mathrm{PN}$ subjects demonstrated a greater increase in step width-to-step length ratio, a greater decrease in step length, and a greater increase in step-time variability than did the control subjects. No subject groupby-environment interactions were identified for step-width variability, step-width range, or step time. Finally, no significant BMI-by-environment or age-by-environment interactions were identified for any of the gait parameters. 

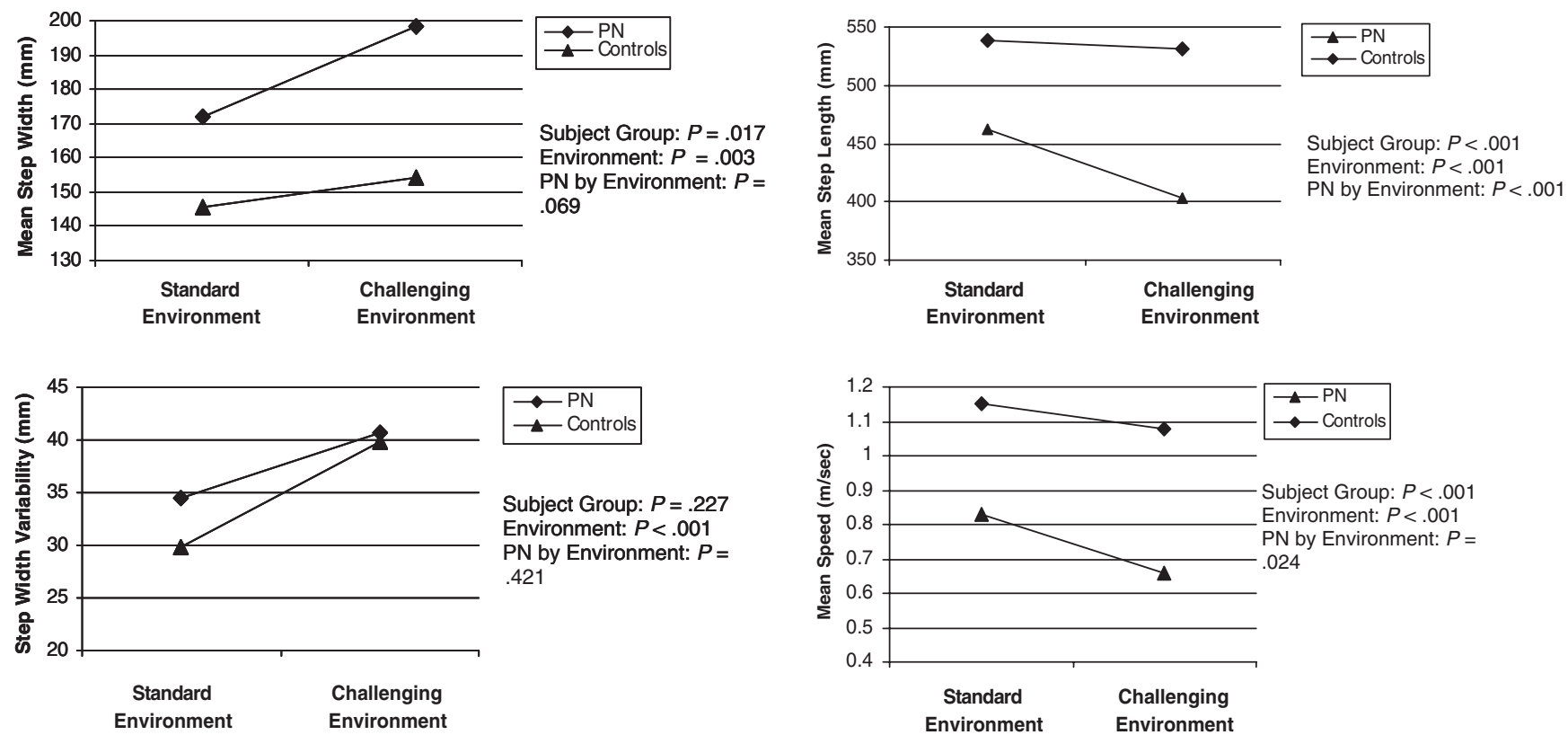

Subject Group: $P=.227$ Environment: $P<.001$ PN by Environment: $P=$ .421
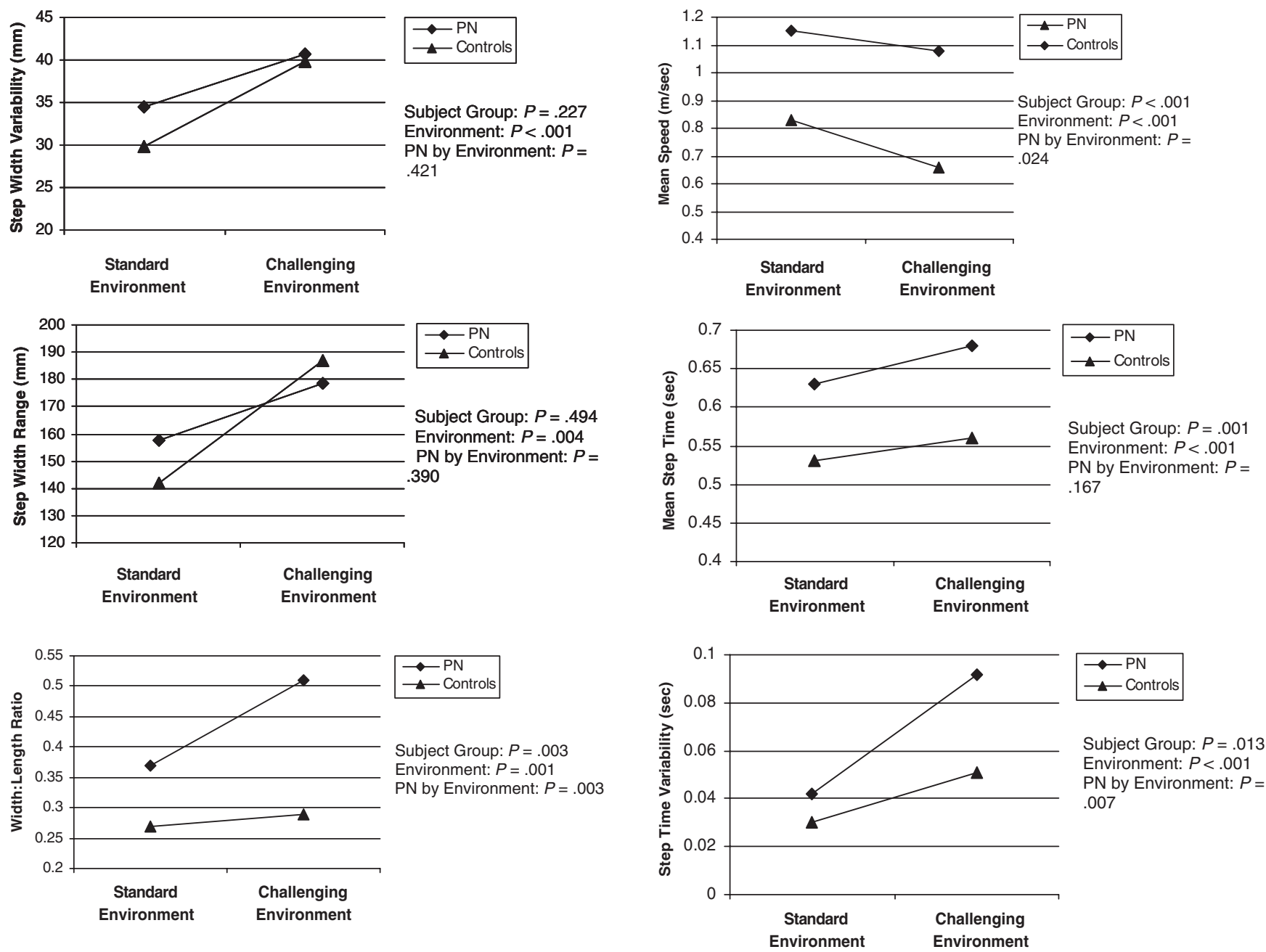

Figure 2. Plots demonstrate interactions between subject group and environment for each gait parameter. Adjacent text box lists main effect significance of subject group and environment and their interaction as determined by repeated measures analysis of variance, adjusting for body mass index and age. $\mathrm{PN}=$ peripheral neuropathy.

\section{Effect of PN Severity}

In the SE, only one gait parameter (mean step width, correlation coefficient $(r)=-0.578 ; P=.049)$ correlated with a clinical measure of PN severity, whereas in the CE, four gait parameters (mean step width, $r=-0.671 ; P=$ .017 ; step-width variability, $r=0.577 ; P=.049$; step-width range, $r=0.675 ; P=.020$; step-time variability, $r=-0.619$; $P=.032)$ did so. Therefore with increasing PN severity, the PN subjects' gait changed minimally in the SE, but in the CE, their gait became narrower and more variable in terms of time and width and steps were taken more quickly.

\section{DISCUSSION}

\section{The Effect of Environment}

The fact that the CE induced significant changes in all gait parameters suggests that its effect on gait was profound. In the CE, steps were slower, wider-based, and more variable in the frontal plane and temporally. These gait changes may have been advantageous to the subjects given that none fell. The slower pace may have allowed better control by decreasing the momentum of the subject's center of mass. The wide-based gait decreases the chance of a collision between swing and stance limbs, a hazard that has been demonstrated 
in older adults when given challenges to their mediolateral stability. ${ }^{17}$

\section{The Effect of Subject Group}

The PN subjects demonstrated slower speed due to shorter step length and longer step time in the SE than the control subjects but no differences in step-width range, step-width variability, or step-time variability. These latter findings suggest that the PN subjects were not less stable than the control subjects in the SE, a finding consistent with that of another study. ${ }^{11} \mathrm{PN}$ subjects demonstrated larger step width-to-step length ratios in the SE than the controls, suggesting a less efficient gait for the former. ${ }^{13}$ Overall, the data suggest that older female PN patients under ideal conditions are able to regulate their gait to a similar degree as healthy women of similar age, but at the cost of speed and efficiency.

In general, the two groups of subjects adapted to the $\mathrm{CE}$ similarly, although the PN subjects often made more extreme adjustments. The most notable exception to this similarity of adaptation was that the PN subjects markedly shortened their step length, but the control subjects did not. The PN subjects simultaneously showed a tendency to increase step width. As a result the PN subjects markedly increased their step width-to-step length ratio and decreased speed, whereas the control subjects changed neither parameter. The PN gait patterns represent a marked contrast to healthy young men and women who adapted to an irregular surface by increasing step time and stride length while maintaining speed. ${ }^{18}$

Why did the PN patients make these gait changes in the $\mathrm{CE}$ ? Widening the base of support during dual stance may have increased stability. Alternatively, or additionally, increasing step width may have loaded the thigh adductors so as to improve frontal plane proprioception and control at the hip. This could compensate for known frontal plane impairments in ankle proprioception and motor function associated with PN. ${ }^{19,20}$ Increasing step width may also have decreased the risk of collision between the swing and stance limbs. Shortening step length may have been necessary to accommodate the step-width increase or to minimize time spent in single limb support in the CE. In support of the latter, previous studies found that PN patients have less ability to maintain one-legged balance on flat and variable surfaces. ${ }^{21,22}$

In the $\mathrm{CE}$, there were no group differences in stepwidth range, and the two groups demonstrated nearly identical step-width variabilities (Table 1 and Figure 2). One way to interpret these data is that these similarly aged groups of women tolerated similar degrees of foot-placement irregularity in the frontal plane in the CE, but the PN subjects needed to slow their speed and increase step widthto-step length ratio, thereby reducing efficiency, to a much greater degree than the control subjects to achieve similar control. With previous evidence that closely links lateral stability and mediolateral foot placement, ${ }^{12,23}$ subject concern for maintenance of dynamic stability in the frontal plane may underlie this strategy.

\section{The Effect of PN Severity}

In the CE, more severe PN was positively associated with step-width variability and range and negatively associated 
with step-time variability and (a trend) step time. One possible explanation for this is that the subjects with more severe PN had a progressive decrease in stability during single limb support in the CE. Consistent with this, previous study identified impairments in neuropathic patients in maintaining balance transferring from bipedal to unipedal stance ${ }^{22}$ and during termination of gait. ${ }^{24}$ Given these limitations, subjects with more severe PN may have needed to take a series of quick successive steps, a common pattern in older persons confronted with a postural perturbation, ${ }^{25}$ leading to reduced step time and step-time variability. The resultant decreased step time gave the subjects with more severe PN less time to modify the trajectory of the swing limb, leading to an increased variability of frontal plane foot placement.

Only one gait parameter demonstrated a significant correlation with PN severity in the SE. In contrast, four gait parameters significantly correlated with $\mathrm{PN}$ in the CE. These findings suggest that studying PN patients under challenging circumstances is more likely to yield insight into gait abnormalities than study under ideal conditions. The absence of a correlation between PN severity and speed suggests that the changes in gait are due to factors other than slowing down.

\section{Implications}

The most effective gait allows a person to move over a variety of terrains safely and with minimal effort. The data suggest that older women with PN are reasonably stable on flat surfaces with good lighting but that their gait speed under these ideal circumstances is somewhat slower than needed in the community for crossing streets with walking signals $\left(0.83 \mathrm{~m} / \mathrm{s}\right.$, or $68 \%$ of the $1.22 \mathrm{~m} / \mathrm{s}$ needed). ${ }^{14}$ When the environment is challenging, the speed of the PN subjects is severely reduced $(0.66 \mathrm{~m} / \mathrm{s}$ or $54 \%$ of the $1.22 \mathrm{~m} / \mathrm{s} \mathrm{nec-}$ essary for crossing streets). Moreover, the accompanying reduction in step length compromises gait efficiency so that PN patients would likely have reduced endurance in the community. Therefore, although PN subjects appear able to navigate irregular surfaces under low light conditions safely, there is a significant cost in terms of speed and efficiency. These patients will likely benefit from a gait aid ${ }^{26}$ and planning when moving in the community.

\section{REFERENCES}

1. Amato AA, Dumitru D. Approach to Peripheral Neuropathy. In: Dumitru D, Amato AA, Zwarts MJ, eds. Electrodiagnostic Medicine. Philadelphia: Hanley \& Belfus, 2002, pp 885-898.

2. Fitzpatrick R, McCloskey DI. Proprioceptive, visual and vestibular thresholds for the perception of sway during standing in humans. J Physiol 1994; 478:173-186.

3. Cavanagh PR, Derr JA, Ulbrecht JS et al. Problems with gait and posture in neuropathic patients with insulin-dependent diabetes mellitus. Diabet Med 1992;9:469-474.
4. Richardson JK, Ching C, Hurvitz EA. The relationship between electromyographically documented peripheral neuropathy and falls. J Am Geriatr Soc 1992;40:1008-1012.

5. Franklin GM, Kahn LB, Baxter J et al. Sensory neuropathy in non-insulindependent diabetes mellitus. The San Luis Valley Diabetes Study. Am J Epidemiol 1990;131:633-643.

6. Harris MI, Flegal KM, Cowie CC et al. Prevalence of diabetes, impaired fasting glucose, and impaired glucose tolerance in U.S. adults: The Third National Health and Nutrition Examination Survey, 1988-94. Diabetes Care 1998; 21:518-524.

7. Berg WP, Alessio HM, Mills EM et al. Circumstances and consequences of falls in independent community-dwelling older adults. Age Ageing 1997; 26:261-268

8. Katoulis EC, Ebdon-Parry M, Lanshammar H et al. Gait abnormalities in diabetic neuropathy. Diabetes Care 1997;20:1904-1907.

9. Courtemanche R, Teasdale N, Boucher P et al. Gait problems in diabetic neuropathic patients. Arch Phys Med Rehab 1996;77:849-855.

10. Mueller MJ, Minor SD, Sahrmann SA et al. Differences in the gait characteristic of patients with diabetes and peripheral neuropathy compared with age-matched controls. Phys Ther 1994;74:299-308.

11. Dingwell JB, Cusumano JP, Sternad D et al. Slower speeds in patients with diabetic neuropathy lead to improved local dynamic stability of continuous overground walking. J Biomech 2000;33:1269-1277.

12. Bauby CE, Kuo AD. Active control of lateral balance in human walking. J Biomech 2000;33:1433-1440.

13. Donelan JM, Kram R, Kuo AD. Mechanical and metabolic determinants of the preferred step width in human walking. Proc R Soc Lond B Biol Sci 2001; 268:1985-1992.

14. Langlois JA, Keyl PM, Guralnik JM et al. Characteristics of older pedestrians who have difficulty crossing the street. Am J Public Health 1997; 87:393-397.

15. Hausdorff JM, Rios DA, Edelberg HK. Gait variability and fall risk in community-living older adults: A 1-year prospective study. Arch Phys Med Rehabil 2001;82:1050-1056.

16. Feldman EL, Stevens MJ, Thomas PK et al. A practical two-step quantitative clinical and electrophysiological assessment for the diagnosis and staging of diabetic neuropathy. Diabetes Care 1994;17:1281-1289.

17. Maki BE, Edmondstone MA, McIlroy WE. Age-related differences in laterally directed compensatory stepping behavior. J Gerontol A Biol Sci Med Sci 2000;55A:M270-M277.

18. Menz HB, Lord SR, Fitzpatrick RC. Acceleration patterns of the head and pelvis when walking on level and irregular surfaces. Gait Posture 2003; 18:35-46.

19. Van den Bosch CG, Gilsing MG, Lee SG et al. Peripheral neuropathy effect on ankle inversion and eversion detection thresholds. Arch Phys Med Rehabil 1995;76:850-856

20. Gutierrez EM, Helber MD, Dealva D et al. Mild diabetic neuropathy affects ankle motor function. Clin Biomech 2001;16:522-528.

21. Ashton-Miller JA, Yeh MW, Richardson JK et al. A cane reduces loss of balance in patients with peripheral neuropathy: Results from a challenging unipedal balance test. Arch Phys Med Rehabil 1996;77:446-452.

22. Richardson JK, Ashton-Miller JA, Lee SG et al. Moderate peripheral neuropathy impairs weight transfer and unipedal balance in the elderly. Arch Phys Med Rehabil 1996;77:1152-1156.

23. MacKinnon CD, Winter DA. Control of whole body balance in the frontal plane during human walking. J Biomech 1993;26:633-644.

24. Meier MR, Desrosiers J, Bourassa P et al. Effect of type II diabetic peripheral neuropathy on gait termination in the elderly. Diabetologia 2001; 44:585-592.

25. Jensen JL, Brown LA, Woollacott MH. Compensatory stepping: The biomechanics of a preferred response among older adults. Exp Aging Res 2001; 27:361-376

26. Richardson JK, Thies S, DeMott T et al. Interventions improve gait regularity in patients with peripheral neuropathy while walking on an irregular surface under low light. J Am Geriatr Soc 2004;52:510-515. 